\title{
Relationship of renal morphology on 3-dimensional ultrasonography with renal pathologic findings and outcome in biopsy-proven nephropathy
}

\author{
JIANWEI XU ${ }^{1}$, SUNG IL HWANG ${ }^{2}$, HAK JONG LEE ${ }^{2}$ and HO JUN CHIN ${ }^{1,3}$ \\ Departments of ${ }^{1}$ Internal Medicine and ${ }^{2}$ Radiology, 12303 Seoul National University Bundang Hospital, \\ Seongnam, Gyeonggi 13620; ${ }^{3}$ Department of Internal Medicine, Seoul National \\ University College of Medicine, Seoul 03080, Republic of Korea
}

Received April 19, 2017; Accepted October 19, 2017

DOI: 10.3892/etm.2017.5626

\begin{abstract}
Kidney morphology has been used to estimate renal functions. The present study investigated the usefulness of kidney length and renal parenchymal volume (RPV) measured using three-dimensional ultrasonography (3-D USG) in estimating renal pathological findings, and the outcome in patients with nephropathy who underwent renal biopsy. In this study, 94 adult patients who had native kidney biopsy with 3-D USG results were included. The mean kidney length and RPV were independent factors of, and positively correlated to the estimated glomerular filtration rate (eGFR). The mean kidney length and RPV had inverse associations with the percentage of global glomerulosclerosis. Higher mean RPV, other than longer kidney length, indicated a lower prevalence of tubular atrophy. During 63.3 \pm 19.3 months of follow-up, a mean RPV of $<125 \mathrm{ml}$ increased the risk of composite outcome by 4.287 fold (95\% confidence interval, 1.133-16.227) as compared with a mean $\mathrm{RPV}$ of $\geq 125 \mathrm{ml}(\mathrm{P}=0.032)$. In conclusion, kidney size was inversely associated with certain nephronal damage and positively associated with eGFR in nephropathy. Furthermore, smaller RPVs predicted worse outcomes of nephropathy.
\end{abstract}

\section{Introduction}

Kidney morphology has been used to predict renal functions in healthy young adults (1). Several studies have also demonstrated the clinical implications of kidney morphology in pathological status. Kidney morphology is related to estimated glomerular filtration rate (eGFR) in patients with chronic kidney disease

Correspondence to: Dr Ho Jun Chin, Department of Internal Medicine, 12303 Seoul National University Bundang Hospital, 300 Gumidong, Bundang-Gu, Seongnam, Kyeongki-Do, Gyeonggi 13620, Republic of Korea

E-mail: mednep@hanmail.net

Key words: glomerulonephritis, glomerulosclerosis, kidney length, outcome, renal parenchymal volume
(CKD) (2-4), in obesity subjects (5), and in patients with nephrectomy due to renal tumor $(6,7)$. Kidney morphology is also related to albuminuria (4) in CKD patients and related to the improvement of renal function after angioplasty in patients with renovascular hypertension (8). These studies suggested that kidney morphology could be closely related to eGFR or proteinuria when the kidney is in a pathological state, In addition, they suggested that the relationship between pathological findings derived from fresh tissues of living patients and kidney morphology could possibly contribute to the explanation of the relationship between kidney morphology and eGFR or between kidney morphology and proteinuria in patients with nephropathy.

Kidney volume and length were usually measured for kidney morphology. However, kidney volume estimated by using computed tomography (CT) predicted eGFR in healthy young adults, whereas kidney length was reported to be not related to eGFR (1). In patients with CKD, kidney volume is a more sensitive indicator of eGFR than kidney length (2). Kidney volume correlates with anthropometric parameters, particularly body surface area (BSA), whereas kidney length only has a weaker relationship with anthropometric parameters (3). Kidney volume is closely correlated to kidney weight $(9,10)$, to which both glomerular number and size show a positive correlation (10). Kidney length shortens with age, while kidney volume is relatively stable $(5,9,11)$. Thus, kidney volume may be a better indicator of eGFR in subjects with or without renal disease.

Contrast-enhanced $\mathrm{CT}$ and magnetic resonance imaging (MRI) are known to provide more accurate measurements of kidney volume than ultrasonography (USG) $(4,6,12)$. However, these techniques are costly and have probable adverse effects in CKD patients due to contrast agents. USG has been improved in terms of both spatial and temporal resolutions to acquire three-dimensional (3-D) images. Three-dimensional USG estimates single kidney GFR in patients with nephrectomy (7) more accurately than two-dimensional USG (13). Renal parenchymal volume (RPV), including the renal cortex and medulla but excluding the renal sinus can be measured by using 3-D USG, repeatedly and non-invasively, at bedside (7), and could be used as an indicator of kidney volume. 
Therefore, we measured kidney length and RPV by using 3-D USG and investigated the efficacy of these parameters to estimate eGFR, proteinuria, renal pathological findings, and the outcomes in patients with nephropathy. We also investigated the relations among kidney morphology, renal pathological findings, and eGFR or proteinuria in patients with nephropathy.

\section{Materials and methods}

Patients. This study was conducted in accordance with the Declaration of Helsinki. It was approved by the Institutional Review Board of Seoul National University Bundang Hospital (IRB no. B-1510/318-111) and was designed as a retrospective cohort study. None of the patients belonged to a vulnerable population or were subject to coercion. As this was a retrospective medical record-based study and the subjects were de-identified, the IRB waived the need for written consent from the patients. We included 99 adult patients who had a kidney biopsy for diagnosis of the cause of proteinuria, hematuria, and/or azotemia, which had persisted for more than 3 months, and had available 3-D USG results from March 2004 to June 2009 . Fifty-three $(53.5 \%)$ of the patients were male and the mean age was $47.3 \pm 14.5$ years old (range, $24-84$ years), whereas forty-six $(46.5 \%)$ of the patients were female and the mean age was $43.5 \pm 18.8$ years (range, 19-87 years). Among these patients, three who had a transplanted kidney biopsy, and two with fewer than 10 glomeruli found in the renal tissue biopsy were excluded. For all of 94 patients included in the analysis, we measured morphological parameters for both kidneys.

Measurements. All data were retrieved through the electronic medical record system, which was approved as a stage-7 hospital information system by the Healthcare Information and Management Systems Society. All the patients were admitted for 2-3 days and had their renal length and volume measured by using 3-D USG before renal biopsy. The patients' heights and weights were measured with the GL-310 weight and height measuring system (GL310; G-Tech International Co., Uijungbu, Kyungki-Do, Korea) on the day of admission. Blood pressures (BP) were measured on the right arm in the seated position by using an automated BP measuring device (HEM-7133; Omron, Kyoto, Japan). We adopted for analysis the morning $\mathrm{BP}$ on the second hospital day before renal biopsy. BSA was calculated as previously described (14). Diabetes mellitus (DM) was defined as the use of anti-diabetic medications, a serum fasting glucose level of $\geq 126 \mathrm{mg} / \mathrm{dl}$ or a glycosylated hemoglobin (HbA1c) level of $\geq 6.5 \%$. Hypertension was defined as a systolic blood pressure of $\geq 140 \mathrm{mmHg}$, a diastolic blood pressure of $\geq 90 \mathrm{mmHg}$ or the use of anti-hypertensive medication. Serum creatinine level was measured by using the modified alkaline picrate Jaffe kinetic method with an automatic analyzer (Toshiba 200FR; Toshiba Medical System Co., Tokyo, Japan). Serum creatinine levels were calibrated to an assay traceable by an isotope dilution mass spectrometry device (Modular P; Roche Diagnostics, Mannheim, Germany). As reported by the manufacturer, the lyophilized calibrator [Roche Calibrator for Automated Systems (CFAS), catalog no. 759350] was prepared with $3.0 \mathrm{ml}$ of Milli-Q water. The
C.F.A.S. calibrator was traceable to reference material SRM $909 \mathrm{~b}$ which was provided by the National Institute of Standards and Technology. The Modular P automatically performed a two-point calibration when there was a reagent lot number change and monitored control values to determine stability of the current calibration. eGFR was calculated by using the CKD-epidemiology collaboration (CKD-EPI) equation (15). On the second hospital day before renal biopsy, morning spot urine samples were collected to measure protein to creatinine ratio (UPCR) in the unit of $\mathrm{g} / \mathrm{g}$ creatinine. We retrieved data on serum creatinine level, UPCR, and complete blood cell counts at admission for renal biopsy, and other data reported within 4 weeks before renal biopsy. We collected complete data for all the patients.

Measurement of renal morphological parameters for both kidneys was conducted with 3-D USG by using an iU-22 ultrasound unit (Philips iU-22; Philips Healthcare, Bothell, WA, USA). The transducer was positioned on the flank region while the patient was in a decubitus position. The maximal length of the kidney was measured in a long-axis view with a 2 to $5 \mathrm{MHz}$ curved array transducer. After measurement of kidney length on the 2-D plane, the 3-D mode was activated and kidney volume data were acquired with a V2-6 broadband curved array transducer. Volume data acquisition scans for each kidney required 3 or 4 sec breath-holds. The acquired volume data were automatically stored and calculated as RPV, excluding volumes of the renal sinus, cysts, or tumors, and using a stacked contour tool installed in the ultrasonography unit (QLAB GI 3DQ Plug-in; Philips Healthcare). Both iU-22 ultrasound and ultrasonography unites were calibrated and manipulated by an experienced expert. The same radiologist measured the morphological parameters for all the patients. Provided that we did not measure the reference values of the kidney length and volume in subjects without renal disease, we used the value reported by Lee et al as the reference value for normal mean kidney length $(10.71 \pm 0.74 \mathrm{~cm})$ measured by using 2-D USG (16) and the value reported by Kim et al as the reference value for normal mean RPV measured by using 3-D USG for Koreans without CKD (175 $\pm 20 \mathrm{ml})(13)$. We defined short kidney length if the length of both kidneys was $<9.5 \mathrm{~cm}$ (approximately less than the mean value- $2 \mathrm{x}$ the standard deviation of length) and a small RPV if the mean volume for both kidneys was $<125 \mathrm{ml}$ (less than the mean value $-2 x$ the standard deviation of volume). We also investigated the medication use before and after the pathological diagnosis. None of the patients received immunosuppressants before renal biopsy.

Pathological diagnosis. All biopsy results were evaluated by using appropriate standards for renal biopsy, including hematoxylin and eosin, periodic acid-Schiff, Masson trichrome, and periodic acid methenamine silver stainings for light microscopy (LM); immunofluorescence (IF) stainings using antibodies against $\operatorname{Ig} \mathrm{A}, \operatorname{IgG}, \operatorname{IgM}, \mathrm{C} 3, \mathrm{C} 1 \mathrm{q}$, and kappa and lambda light chains; and electron microscopic examinations, as previously described (17). The pathological diagnosis was classified as either a nephritic or a non-nephritic lesion. One pathologist reviewed the pathologic findings. Pathological findings, which were graded semi-quantitatively, were graded from normal to severe (absence of lesion, focal mild changes: 
Presence of the lesion in $<25 \%$ of results, focal moderate changes: Presence of the lesion in 25-50\% of results, focal marked or diffuse changes: Presence of the lesion in $>50 \%$ of results). The patients were also grouped according to the presence or absence of a lesion.

Outcome. The primary outcome was the composite of death from any cause and/or progression to end stage renal disease (ESRD) that required renal replacement therapy after renal biopsy. Mortality data, available since 1992, were retrieved from the Korean National Statistical Office; and ESRD progression data, available since 1986, were retrieved from the Korean ESRD registry. We combined all of these data together.

Statistics. The data are shown as mean \pm SD. All the analyses were conducted by using SPSS Statistics version 22.0 (IBM Corp., Armonk, NY, USA). The correlations between eGFR, UPCR, or pathological findings and kidney length or volume were assessed by using the Pearson's coefficient. Multivariate analyses to detect factors independently related to continuous and binomial categorical variables, were performed by using linear and logistic regression analyses, respectively, adjusted for related factors. Differences in continuous variables were analyzed by using two-tailed, unpaired Student's t-tests or one-way analysis of variance according to the number of subgroups. By contrast, differences in categorical variables were analyze by using Chi-square tests or the Fisher's exact test according to the number of cases in each cell. We analyzed the cumulative incidence of the outcome between the patients categorized into groups according to a mean kidney length of $9.5 \mathrm{~cm}$ or the mean RPV of both kidneys of $125 \mathrm{ml}$, by using the Kaplan-Meier analysis. Incidence rates were compared by using the log-rank test. The receiver-operator curve (ROC) was also used to identify the cutoff value of mean kidney length or the mean RPV of both kidneys in our cohort and Youden's index was applied for the selection of the best cutoff value. We also analyzed the cumulative incidence of the outcome between the patients categorized into groups according to the best cutoff value by using the Kaplan-Meier analysis. To determine whether renal morphological data were independently related to the incidence of the outcome, we used the Cox hazard proportional analysis. Two-sided P-values were reported with 0.05 selected as the level of statistical significance.

\section{Results}

Patient characteristics. Forty-two of the patients were men (44.7\%), and the mean age was $45.5 \pm 16.4$ years (range, 19-84 years; Table I). Of the patients, $56.4 \%$ (53/94), $17.0 \%$ $(16 / 94)$, and $26.6 \%(25 / 94)$ had eGFRs of $\geq 90,60-89$, $<60 \mathrm{ml} /\left(\min \cdot 1.73 \mathrm{~m}^{2}\right)$, respectively. The mean UPCR was $3.88 \mathrm{~g} / \mathrm{g}$ of $\mathrm{Cr}$, and $43.6 \%$ (41/94) of the patients had an UPCR $\geq 3.00 \mathrm{~g} / \mathrm{g}$ Cr. The diagnosis was nephritic in 50 patients and 44 non-nephritic lesion in 44 patients. The most common pathological diagnosis was IgA nephropathy (36.2\%), followed by minimal change lesion (9.6\%) and membranous nephropathy (8.5\%; Table I). Pathological findings are described in Table II. The mean length and parenchymal volume of both kidneys were $10.6 \pm 0.9 \mathrm{~cm}$ and $138.3 \pm 34.6 \mathrm{ml}$, respectively. No significant difference in mean kidney length or RPV was found
Table I. Basal characteristics of the patient with renal biopsy.

\begin{tabular}{|c|c|}
\hline Parameters & Values \\
\hline Age (years) & $45.5 \pm 16.4$ \\
\hline Male & $42(44.7)$ \\
\hline Height (cm) & $163 \pm 9$ \\
\hline Weight (kg) & $63.3 \pm 13$ \\
\hline BMI $\left(\mathrm{kg} / \mathrm{m}^{2}\right)^{\mathrm{a}}$ & $23.8 \pm 3.9$ \\
\hline $\operatorname{BSA}\left(\mathrm{m}^{2}\right)^{\mathrm{a}}$ & $1.68 \pm 0.2$ \\
\hline $\mathrm{SBP}(\mathrm{mmHg})^{\mathrm{a}}$ & $121 \pm 17$ \\
\hline $\mathrm{DBP}(\mathrm{mmHg})^{\mathrm{a}}$ & $69 \pm 12$ \\
\hline Creatinine (mg/dl) & $1.43 \pm 1.78$ \\
\hline $\mathrm{eGFR}\left(\mathrm{ml} / \mathrm{min} / 1.73 \mathrm{~m}^{2}\right)^{\mathrm{a}}$ & $84.8 \pm 38.9$ \\
\hline UPCR $(\mathrm{g} / \mathrm{g} \mathrm{cr})^{\mathrm{a}}$ & $3.88 \pm 5.33$ \\
\hline Diabetes mellitus & $14(14.9)$ \\
\hline Hypertension & $62(66)$ \\
\hline \multicolumn{2}{|l|}{ Total kidney } \\
\hline Mean length $(\mathrm{cm})^{\mathrm{a}}$ & $10.6 \pm 0.9$ \\
\hline Mean RPV $(\mathrm{ml})^{\mathrm{a}}$ & $138.3 \pm 34.6$ \\
\hline Left kidney & \\
\hline Length $(\mathrm{cm})^{\mathrm{a}}$ & $10.6 \pm 1.1$ \\
\hline $\mathrm{RPV}(\mathrm{ml})^{\mathrm{a}}$ & $141.8 \pm 37.1$ \\
\hline Right kidney & \\
\hline Length $(\mathrm{cm})^{\mathrm{a}}$ & $10.6 \pm 0.9$ \\
\hline $\mathrm{RPV}(\mathrm{ml})^{\mathrm{a}}$ & $134.7 \pm 35.8$ \\
\hline \multicolumn{2}{|l|}{ Urinalysis } \\
\hline Albumin negative & $15(16)$ \\
\hline Trace & $5(5.3)$ \\
\hline $1+$ & $5(2.3)$ \\
\hline $2+$ & $19(20.2)$ \\
\hline$\geq 3+$ & $50(53.2)$ \\
\hline \multicolumn{2}{|l|}{$\mathrm{RBC}^{\mathrm{a}}$} \\
\hline$<5 / \mathrm{HPF}^{\mathrm{a}}$ & $31(33)$ \\
\hline 5-9/HPF & $12(12.8)$ \\
\hline$\geq 10 / \mathrm{HPF}$ & $51(54.3)$ \\
\hline \multicolumn{2}{|l|}{$\mathrm{WBC}^{\mathrm{a}}$} \\
\hline$<5 / \mathrm{HPF}$ & $72(76.6)$ \\
\hline 5-9/HPF & $10(10.6)$ \\
\hline$\geq 10 / \mathrm{HPF}$ & $12(12.8)$ \\
\hline Immunosuppressant $^{\mathrm{a}}$ & $35(37.2)$ \\
\hline Steroid & $33(35.1)$ \\
\hline Cyclophosphamide & $14(14.9)$ \\
\hline Others $^{\mathrm{a}}$ & $17(18.1)$ \\
\hline
\end{tabular}

Pathologic diagnosis ${ }^{\mathrm{a}}$

Non-nephritic

AIN

$2(2.1)$

Alport's syndrome $1(1.1)$

Amyloidosis $3(3.2)$

C1q nephropathy $3(3.2)$

CIN

$3(3.2)$

Diabetic nephropathy $3(3.2)$

FSGS

$6(6.4)$

$\begin{array}{ll}\text { Hypertensive } & 2(2.1) \\ \text { MCD } & 9(9.6)\end{array}$ 
Table I. Continued.

\begin{tabular}{lc}
\hline Parameters & Values \\
\hline Myeloma kidney & $1(1.1)$ \\
MN & $8(8.5)$ \\
Thin membrane disease & $3(3.2)$ \\
Nephritic & \\
Crescentic & \\
GN & $3(3.2)$ \\
DPLN & $3(3.2)$ \\
HSN & $1(1.1)$ \\
IgA nephritis & $34(36.2)$ \\
MPGN & $2(2.1)$ \\
Non-specific GN & $6(6.4)$ \\
PSGN & $1(1.1)$
\end{tabular}

Values are presented as the mean \pm standard deviation or the number of patients with the corresponding percentage. ${ }^{\mathrm{a} B M I}$, body mass index; SBP, systolic blood pressure; BSA, body surface area; DBP, diastolic blood pressure; eGFR, calculated by Chronic Kidney Disease Epidemiology Collaboration (CKD-EPI) equation using isotope-dilution mass spectrometry (IDMS) traceable creatinine; UPCR, urine protein to creatinine ratio in $\mathrm{g} / \mathrm{g}$ creatinine unit, length: The longest length of kidney; RPV, renal parenchymal volume, Mean length: Mean length of both kidneys; Mean RPV, mean parenchymal volume of both kidneys; RBC, red blood cell; HPF, high power-field under 400-fold magnification of light microscopy; WBC, white blood cell, immunosuppressant: Medication after renal biopsy during follow-up period, including steroid, cyclophosphamide, mycophenolate, cyclosporine, tacrolimus, or azathioprine, others: Immunosuppressant including mycophenolate, cyclosporine, tacrolimus, or azathioprine; AIN, acute interstitial nephritis; CIN, chronic interstitial nephritis; FSGS, focal segmental glomerulosclerosis; MCD, minimal change disease; MN, membranous nephropathy; GN, glomerulonephritis; DPLN, diffuse proliferative lupus nephritis, lupus nephritis class IV defined by International Society of Nephrology/Renal Pathology Society (ISN/RPS) classification; HSN, Henoch-Schölein nephritis; MPGN, membranoproliferative nephritis; PSGN, post-streptococcal glomerulonephritis.

between the left and right kidneys. The mean RPV showed significant correlations with weight, height, body mass index (BMI), and BSA in 94 subjects. The mean kidney length also showed significant correlations with weight, height, and BMI, but weaker correlations compared with those of the mean RPV (Table III). In the multiple linear regression analysis, height was the only independent factor related to mean kidney length $(\mathrm{B}=0.026, \mathrm{P}<0.001)$; and $\mathrm{BSA}$ was related to mean $\mathrm{RPV}$ $(\mathrm{B}=77.750, \mathrm{P}<0.001)$.

Renal function, kidney morphology and pathologic findings. We analyzed the relationship between kidney morphology and renal function by using multiple linear regression analysis adjusted for clinical parameters (Table IV). Both mean kidney length and RPV were identified as independent factors with positive correlations with eGFR level. However, they were not related to UPCR. The pathological findings related to eGFR were percent of global glomerulosclerosis $(\mathrm{P}=0.037)$ and grade of interstitial inflammation $(\mathrm{P}<0.001)$ in the multiple
Table II. Pathologic findings of renal biopsy.

\begin{tabular}{|c|c|}
\hline Pathologic findings & Values \\
\hline No. of glomerulus & $48.3 \pm 22.6$ \\
\hline Global glomerulosclerosis (\%) & $17.1 \pm 22.8$ \\
\hline Segmental glomerulosclerosis (\%) & $5.3 \pm 9.7$ \\
\hline Glomerular crescent $(\%)$ & $0.2 \pm 0.4$ \\
\hline Glomerular necrosis (\%) & $0.0 \pm 0.2$ \\
\hline Glomerular ischemia (\%) & $0.1 \pm 0.3$ \\
\hline \multicolumn{2}{|l|}{ Glomerular hypertrophy (\%) } \\
\hline None & 59.6 \\
\hline Mild & 31.9 \\
\hline Moderate & 7.4 \\
\hline Severe & 1.1 \\
\hline \multicolumn{2}{|l|}{ Glomerular cellularity (\%) } \\
\hline None & 29.8 \\
\hline Mild & 55.3 \\
\hline Moderate & 10.6 \\
\hline Severe & 4.3 \\
\hline \multicolumn{2}{|l|}{ Mesangial proliferation (\%) } \\
\hline None & 27.7 \\
\hline Mild & 17 \\
\hline Moderate & 41.5 \\
\hline Severe & 13.8 \\
\hline \multicolumn{2}{|l|}{ Interstitial fibrosis (\%) } \\
\hline None & 26.6 \\
\hline Mild & 50 \\
\hline Moderate & 13.8 \\
\hline Severe & 9.6 \\
\hline \multicolumn{2}{|l|}{ Interstitial inflammation (\%) } \\
\hline None & 26.6 \\
\hline Mild & 48.9 \\
\hline Moderate & 11.7 \\
\hline Severe & 12.8 \\
\hline \multicolumn{2}{|l|}{ Tubular atrophy (\%) } \\
\hline None & 24.5 \\
\hline Mild & 50 \\
\hline Moderate & 12.8 \\
\hline Severe & 12.8 \\
\hline \multicolumn{2}{|l|}{ Vascular lesion (\%) } \\
\hline Absence & 60.6 \\
\hline Presence & 39.4 \\
\hline
\end{tabular}

Values are presented as the mean \pm standard deviation or percentage. One pathologist counted the percentages of glomeruli with global sclerosis, segmental sclerosis, crescent, ischemic changes, or necrosis, and assessed the renal changes of glomerular hypertrophy, glomerular cellularity, mesangial proliferation, interstitial fibrosis, interstitial inflammation, and tubular atrophy, semi-quantitatively. We scored from normal to severe form (absence of lesion, focal mild changes: Presence of the lesion in $<25 \%$ of results, focal moderate changes: Presence of the lesion in $25-50 \%$ of results, focal marked or diffuses changes: Presence of the lesion in $>50 \%$ of results). We assessed the arteriolar hyalinosis and arteriosclerosis defined as non-hyaline thickening of the vascular wall with reduction of the lumen. We grouped the arteriolar lesion as presence or absence. 
Table III. Correlation between anthropometric parameters and kidney size.

\begin{tabular}{|c|c|c|c|c|}
\hline \multirow[b]{2}{*}{ Parameter } & \multicolumn{2}{|c|}{ Mean kidney length } & \multicolumn{2}{|c|}{ Mean RPV } \\
\hline & $\mathrm{r}^{\mathrm{a}}$ & P-value & $\mathrm{r}^{\mathrm{a}}$ & P-value \\
\hline Age & -0.043 & 0.681 & -0.073 & 0.485 \\
\hline Gender & -0.005 & 0.962 & 0.182 & 0.080 \\
\hline Weight & 0.249 & 0.016 & 0.430 & $<0.001$ \\
\hline Height & 0.257 & 0.013 & 0.366 & $<0.001$ \\
\hline BMI & 0.274 & 0.007 & 0.455 & $<0.001$ \\
\hline BSA & 0.140 & 0.179 & 0.285 & 0.005 \\
\hline
\end{tabular}

${ }^{a}$ r, Pearson's correlation coefficient; RPV, renal parenchymal volume; BMI, body mass index; BSA, body surface area.

Table IV. The correlation between morphometric measurements of kidney and eGFR or proteinuria.

\begin{tabular}{lccccc}
\hline Measurement & $\mathrm{B}$ & $\mathrm{t}$ & $95 \%$ CI for B & P-value \\
\hline $\begin{array}{l}\text { For eGFR } \\
\text { Mean kidney }\end{array}$ & 11.601 & 4.082 & 5.941 & 17.261 & $<0.001$ \\
$\begin{array}{l}\text { length }(\mathrm{cm}) \\
\text { Mean RPV }(10 \mathrm{ml})\end{array}$ & 1.895 & 2.250 & 0.022 & 0.357 & 0.027 \\
$\begin{array}{l}\text { For UPCR } \\
\text { Mean kidney }\end{array}$ & uc & -1.042 & -0.110 & 0.848 & 0.300 \\
$\begin{array}{l}\text { length }(\mathrm{cm}) \\
\text { Mean RPV }(10 \mathrm{ml})\end{array}$ & uc & 1.722 & 0.181 & 0.905 & 0.089
\end{tabular}

aeGFR, calculated by the Chronic Kidney Disease Epidemiology Collaboration (CKD-EPI) equation using isotope-dilution mass spectrometry (IDMS) traceable creatinine; eGFR, estimated glomerular filtration rate. UPCR, urine protein to creatinine ratio in $\mathrm{g} / \mathrm{g}$ creatinine unit; RPV, renal parenchymal volume; uc, unable to calculate; $\mathrm{CI}$, confidence interval. Multivariate linear regression analysis was applied to reveal the correlation between eGFR and morphometric measurement, adjusted for age, sex, presence of diabetes mellitus and hypertension, systolic blood pressure, levels of hemoglobin, serum C-reactive protein, phosphorus, uric acid, and presence of hematuria To reveal the correlation between UPCR and morphometric measurement, it was adjusted for age, sex, presence of diabetes mellitus, levels of serum albumin, bilirubin, cholesterol, glucose, phosphorus, and uric acid, which were related factors to eGFR or UPCR.

linear regression analysis adjusted for interstitial fibrosis, tubular atrophy, and pathologic diagnosis. For the UPCR, the only independent pathological factor was percent of global glomerulosclerosis $(\mathrm{P}=0.050)$ adjusted for pathological diagnosis.

Mean kidney length and RPV were inversely related to percent of global glomerulosclerosis, grades of interstitial fibrosis and inflammation, and tubular atrophy (Table V). For each pathological finding, the multivariate analysis revealed that mean kidney length and RPV had inverse relationships with percent of global glomerulosclerosis (Table VI). These
Table V. The correlation coefficients between renal morphology and pathologic findings.

\begin{tabular}{lcc}
\hline Characteristic & $\begin{array}{c}\text { Mean kidney } \\
\text { length }(\mathrm{cm})\end{array}$ & $\begin{array}{c}\text { Mean } \\
\text { RPV (ml) }\end{array}$ \\
\hline Glomerular hypertrophy (grade) & 0.065 & 0.109 \\
Global glomerulosclerosis (\%) & $-0.489^{\mathrm{a}}$ & $-0.392^{\mathrm{a}}$ \\
Segmental glomerulosclerosis (\%) & -0.018 & -0.106 \\
Glomerular crescent (\%) & 0.151 & 0.086 \\
Glomerular necrosis (\%) & 0.035 & -0.064 \\
Glomerular ischemia (\%) & -0.028 & 0.030 \\
Glomerular cellularity (grade) & 0.104 & -0.044 \\
Mesangial proliferation (grade) & 0.084 & -0.030 \\
Interstitial fibrosis (grade) & $-0.340^{\mathrm{b}}$ & $-0.269^{\mathrm{b}}$ \\
Interstitial inflammation (grade) & $-0.318^{\mathrm{b}}$ & $-0.302^{\mathrm{b}}$ \\
Tubular atrophy (grade) & $-0.341^{\mathrm{b}}$ & $-0.319^{\mathrm{b}}$ \\
Vascular lesion & 0.070 & 0.015 \\
(presence or absence) & & \\
\hline
\end{tabular}

${ }^{\mathrm{a}} \mathrm{P}<0.001,{ }^{\mathrm{b}} \mathrm{P}<0.01 ; \mathrm{RPV}$, renal parenchymal volume. One pathologist counted the percentage of glomeruli with global sclerosis, segmental sclerosis, crescent, ischemic changes, or necrosis, and assessed the renal changes of glomerular hypertrophy, glomerular cellularity, mesangial proliferation, interstitial fibrosis, interstitial inflammation, and tubular atrophy, semi-quantitatively. We scored the findings from normal to severe (absence of the lesion, focal mild changes: Less than $25 \%$ are affected by the lesion, focal moderate changes: $25-50 \%$ are affected, focal marked or diffuse changes: $50 \%$ or more are affected). We assessed the arteriolar hyalinosis and arteriosclerosis defined as non-hyaline thickening of the vascular wall with reduction of the lumen. We grouped the arteriolar lesion as presence or absence.

relationships were also evident in patients with or without nephritis (data not shown). No collinearity was found between eGFR, another independent factor of global glomerulosclerosis, and mean kidney length or RPV (variance inflation factor $=1.108$ and 1.020 , respectively) when we estimated percent of global glomerulosclerosis by using multivariate analysis. Higher mean RPV, but not kidney length, indicated lower prevalence of tubular atrophy in multivariate analysis.

Kidney morphology and the outcome . During 63.3 \pm 19.3 months of follow-up, the disease progressed to ESRD in five patients, all of whom were alive at the end of the follow-up period. Six deaths, including two cases of cancers, one case of chronic obstructive lung disease, one case of glomerulonephritis, one case of chronic renal failure, and one case of accident were observed. The ROC analysis indicated that best cutoff point of mean kidney length and mean RPV was $9.4 \mathrm{~cm}$ and $120.7 \mathrm{ml}$, respectively. Patients with small mean RPVs of $<125 \mathrm{ml}$ tended to have a higher incidence rate of ESRD or mortality (Fig. 1), and showed a higher rate of composite outcome by Fisher exact test and log-rank test (Figs. 1 and 2). Patients with small mean RPVs of $<120.7 \mathrm{ml}$ displayed a higher incidence rate of ESRD (Fig. 1) and composite outcome by Fisher's exact test and log-rank test (Figs. 1 and 2). With the Cox proportional hazard model, the mean RPV of $<125 \mathrm{ml}$ 
Table VI. Multivariate analysis for factors related to pathologic findings of renal biopsy.

\begin{tabular}{|c|c|c|c|c|c|c|c|c|}
\hline \multirow{2}{*}{$\frac{\text { Pathology }}{\text { Parameter }}$} & \multicolumn{2}{|c|}{$\begin{array}{c}\text { Glomerular } \\
\text { sclerosis }(\%)\end{array}$} & \multicolumn{2}{|c|}{$\begin{array}{l}\text { Interstitial fibrosis } \\
\text { (presence) }\end{array}$} & \multicolumn{2}{|c|}{$\begin{array}{c}\text { Interstitial } \\
\text { inflammation } \\
\text { (presence) }\end{array}$} & \multicolumn{2}{|c|}{$\begin{array}{l}\text { Tubular atrophy } \\
\text { (presence) }\end{array}$} \\
\hline & t-value & P-value & OR & P-value & OR & P-value & OR & P-value \\
\hline \multicolumn{9}{|l|}{ Model $1^{\mathrm{a}}$} \\
\hline Mean kidney length & -3.819 & $<0.001$ & uc & 0.135 & uc & 0.271 & uc & 0.181 \\
\hline Mean RPV & -3.939 & $<0.001$ & uc & 0.087 & uc & 0.364 & 0.983 & 0.026 \\
\hline \multicolumn{9}{|l|}{ Model $2^{\mathrm{b}}$} \\
\hline Mean kidney length & -3.600 & 0.001 & uc & 0.682 & uc & 0.984 & uc & 0.938 \\
\hline Mean RPV & -3.506 & 0.001 & uc & 0.267 & uc & 0.540 & 0.977 & 0.016 \\
\hline
\end{tabular}

RPV, renal parenchymal volume; OR, Odds ratio; uc, unable to calculate. ${ }^{a}$ Model 1 , all analyses were adjusted for age, sex, presence of diabetes mellitus and hypertension, eGFR, and UPCR. ${ }^{b}$ Model 2, multivariate analyses were analyzed with adjustment for the factors related to each pathologic finding. For the percent of glomerular sclerosis, the multiple linear regression analysis was adjusted for the presence of diabetes mellitus, serum levels of albumin, complement C4, cholesterol, eGFR, hemoglobin, and uric acid, and morphologic data measured by ultrasonography. For the presence of interstitial fibrosis, the multiple logistic regression analysis was adjusted for the presence of hypertension, serum bilirubin more than $0.6 \mathrm{mg} / \mathrm{dl}$, serum level of complement $\mathrm{C} 4$, cholesterol, eGFR, hemoglobin, protein, uric acid, presence of pyuria, and morphologic data measured by ultrasonography, and, for the presence of interstitial inflammation, adjusted for age, presence of hypertension, serum bilirubin more than $0.6 \mathrm{mg} / \mathrm{dl}$, serum levels of complement $\mathrm{C} 4$, cholesterol, C-reactive protein, eGFR, GOT, hemoglobin, protein, uric acid, level of WBC, presence of hematuria and albuminuria $\geq 3+$ by a dipstick test, and morphologic data measured by ultrasonography, and, for the presence of tubular atrophy, adjusted for age, presence of hypertension, serum bilirubin more than $0.6 \mathrm{mg} / \mathrm{dl}$, serum levels of complement C4, cholesterol, C-reactive protein, eGFR, GOT, hemoglobin, protein, uric acid, level of WBC, and morphologic data measured by ultrasonography.
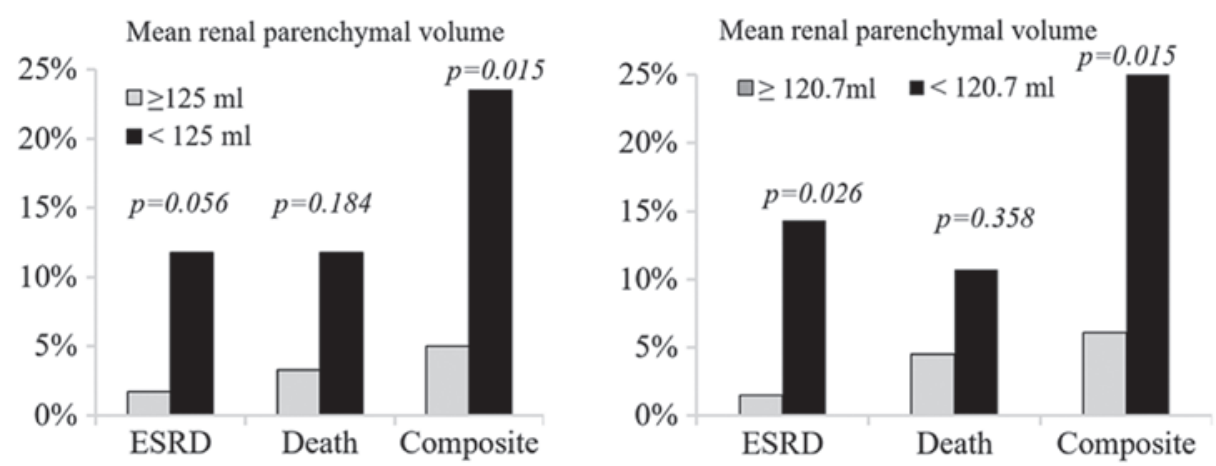

Figure 1. The incidence of outcomes according to mean renal parenchymal volume. The P-value was estimated by using the Fisher's exact test. Sixty patients had a mean parenchymal volume of $\geq 125 \mathrm{ml}$, and 34 patients had a mean parenchymal volume of $<125 \mathrm{ml}$ whereas, sixty-six patients had a mean parenchymal volume of $\geq 120.7 \mathrm{ml}$, and 28 patients had a mean parenchymal volume of $<120.7 \mathrm{ml}$.

increased the risk of composite outcome by 4.287 -fold [95\% confidence interval (CI): 1.133-16.227] as compared with the mean $\mathrm{RPV} \geq 125 \mathrm{ml}(\mathrm{P}=0.032$; Table VII), whereas, the mean $\mathrm{RPV}$ of $<120.7 \mathrm{ml}$ increased the risk of composite outcome by 3.395 -fold (95\% CI: $0.985-11.6)$ as compared with the mean $\mathrm{RPV} \geq 120.7 \mathrm{ml}$ ( $\mathrm{P}=0.053$; Table VIII). However. In a fully-adjusted model which adjusted with mean RPV categorized by $125 \mathrm{ml}$ and $120.7 \mathrm{ml}$, the mean $\mathrm{RPV}$ of $<125 \mathrm{ml}$, other than the mean RPV of $<120.7 \mathrm{ml}(\mathrm{P}=0.882)$, was a risk factor for the composite outcome (hazard ratio: $4.287,95 \%$ CI: $1.133-16.227, \mathrm{P}=0.032)$. Interaction terms were created between mean RPV and other risk factors (eGFR, grade of interstitial fibrosis, tubular atrophy, and percent of glomerulosclerosis in renal biopsy findings) to identify significant interactions. The only interaction term that was significantly associated with the composite outcome was mean RPV $x$ percent of glomerulosclerosis $(\mathrm{P}=0.023)$. Another factor independently associated with the composite outcome was eGFR $(\mathrm{P}=0.002)$.

\section{Discussion}

In this study, kidney morphological parameters were obtained by using 3-D USG, which was more accurate and robust than 2-D USG, and cheaper and less harmful than MRI. 3-D volumetry has lower mean absolute deviation in related to the gold-standard measurements (the fluid displacement method provided the 'gold standard') in assessing the volume of human 
Table VII. The relationship between morphometric measurements of the kidney on the outcome of patients with nephropathy (reference values were obtained from subjects without renal disease).

\begin{tabular}{|c|c|c|c|c|c|c|c|}
\hline \multirow{3}{*}{$\begin{array}{l}\text { Morphometric variables }^{\mathrm{a}} \\
\text { Mean kidney length }\end{array}$} & \multicolumn{2}{|c|}{ Model $1^{\mathrm{a}}$} & \multicolumn{5}{|c|}{ Model $2^{b}$} \\
\hline & \multirow{2}{*}{$\begin{array}{l}\mathrm{B} \\
\mathrm{uc}\end{array}$} & \multirow{2}{*}{$\frac{\text { P-value }}{0.381}$} & \multirow{2}{*}{ B } & \multirow{2}{*}{$\begin{array}{c}\mathrm{RR} \\
\mathrm{nd}\end{array}$} & \multicolumn{2}{|c|}{$95 \%$ CI of RR } & \multirow{2}{*}{$\frac{\text { P-value }}{\text { nd }}$} \\
\hline & & & & & nd & nd & \\
\hline Group of length $(<9.5 \mathrm{~cm})$ & uc & 0.057 & nd & nd & nd & nd & nd \\
\hline Mean RPV & uc & 0.204 & nd & nd & nd & nd & nd \\
\hline Group of RPV $(<125 \mathrm{ml})$ & 1.623 & 0.017 & 1.456 & 4.287 & 1.133 & 16.227 & 0.032 \\
\hline
\end{tabular}

RR, relative risk; RPV, renal parenchymal volume; uc, unable to calculate; nd, not done; CI, confidence interval. ${ }^{a}$ Model 1 , Cox's proportional hazard model for the outcome with single morphometric variable. ${ }^{b}$ Model 2, Cox's proportional hazard model for the outcome with each significant variable in model 1 and the other related factors, such as estimated glomerular filtration rate, hemoglobin, presence of hematuria and proteinuria $\geq 3+$ by a dipstick test, grade of interstitial fibrosis, tubular atrophy and percent of glomerulosclerosis on renal biopsy findings, which were univariate factors to the outcome, age, and sex. Group of length: Patients grouped by the criterion of mean length of both kidneys $9.5 \mathrm{~cm}$, Group of RPV: Patients grouped by the criterion of mean parenchymal volume of both kidneys $125 \mathrm{ml}$.

Table VIII. The relationship between morphometric measurements of the kidney on the outcome of patients with nephropathy (optimal cutoff values from ROC-curve analysis were used as reference values).

\begin{tabular}{|c|c|c|c|c|c|c|c|}
\hline \multirow{3}{*}{$\begin{array}{l}\text { Morphometric variables }^{\mathrm{a}} \\
\text { Mean kidney length }\end{array}$} & \multicolumn{2}{|c|}{ Model $1^{\mathrm{a}}$} & \multicolumn{5}{|c|}{ Model $2^{\mathrm{b}}$} \\
\hline & \multirow{2}{*}{ B } & \multirow{2}{*}{$\frac{\text { P-value }}{0.381}$} & \multirow{2}{*}{$\frac{\mathrm{B}}{\mathrm{nd}}$} & \multirow{2}{*}{$\begin{array}{l}\mathrm{RR} \\
\mathrm{nd}\end{array}$} & \multicolumn{2}{|c|}{$95 \% \mathrm{CI}$ of RR } & \multirow{2}{*}{$\frac{\text { P-value }}{\text { nd }}$} \\
\hline & & & & & nd & nd & \\
\hline Group of length $(<9.4 \mathrm{~cm})$ & uc & 0.047 & uc & uc & uc & uc & 0.216 \\
\hline Mean RPV & uc & 0.204 & nd & nd & nd & nd & nd \\
\hline Group of RPV $(<125.7 \mathrm{ml})$ & 1.483 & 0.018 & 1.222 & 3.395 & 0.985 & 11.6 & 0.053 \\
\hline
\end{tabular}

RR, relative risk; RPV, renal parenchymal volume; uc, unable to calculate; nd, not done. ${ }^{\text {aModel }} 1$, Cox's proportional hazard model for the outcome with single morphometric variable. ${ }^{b}$ Model 2, Cox's proportional hazard model for the outcome with each significant variable in model 1 and the other related factors, such as estimated glomerular filtration rate, hemoglobin, presence of hematuria and proteinuria $\geq 3+$ by a dipstick test, grade of interstitial fibrosis, tubular atrophy and percent of glomerulosclerosis on renal biopsy findings, which were univariate factors to the outcome, age, and sex. Group of length: Patients grouped by the criterion of mean length of both kidneys $9.4 \mathrm{~cm}$, Group of RPV: Patients grouped by the criterion of mean parenchymal volume of both kidneys $120.7 \mathrm{ml}$.

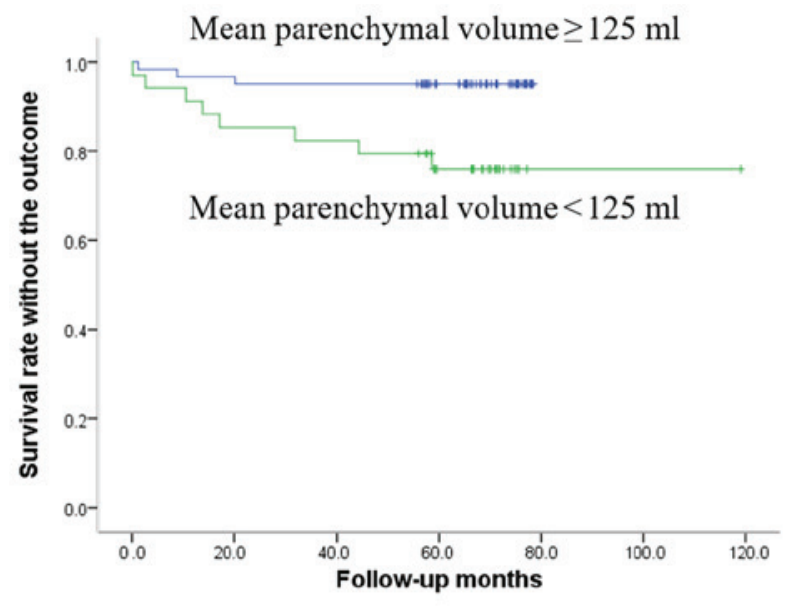

$P$-value by log-rank test $=0.008$

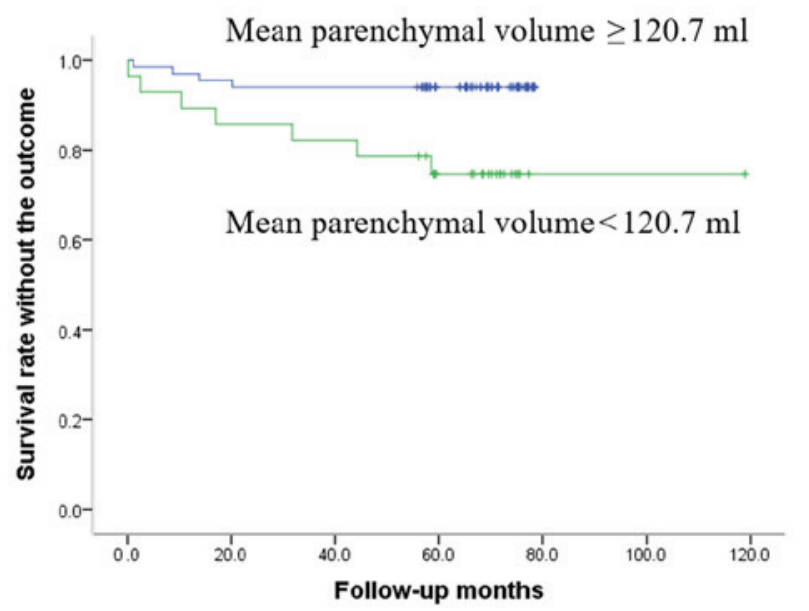

$P$-value by log-rank test $=0.010$

Figure 2. The results of the survival analysis of the outcomes according to the mean renal parenchymal volume of both kidneys. Patients with small mean $\mathrm{RPVs}$ of $<125 \mathrm{ml}$ showed a higher incidence rate composite outcome by log-rank test as compared with patients with small mean RPVs of $\geq 125 \mathrm{ml}$. Patients with small mean RPVs of $<120.7 \mathrm{ml}$ displayed a higher incidence rate composite outcome by log-rank test as compared with patients with small mean RPVs of $\geq 120.7 \mathrm{ml}$. RPV, renal parenchymal volume. 
cadaver kidneys as compared with 2-D images, and also showed a satisfactory concordance correlation to gold-standard measurement (18). Volume measurements obtained from MRI with the voxel-count method showed little deviation from the true renal volume (gold-standard) (19). Kidney volume measured by 3-D USG and MRI yielded a good correlation $(r=0.82, P<0.001)$ and the accuracy of $3-D$ USG was $91.6 \pm 8.4 \%$ in relation to MRI volumes of human kidneys (20). Therefore, 3-D USG performs high accuracy in renal volume estimation for clinical applications. Kidney size, particularly RPV, was inversely related to certain nephronal damage presenting as global glomerulosclerosis and tubular atrophy, whereas percent of global glomerulosclerosis $(\mathrm{P}=0.037)$ was inversely related to eGFR. This could be a pathophysiological mechanism to explain a positive relationship between renal morphology and eGFR, regardless of age, sex, and anthropometric characteristics. Furthermore, we found that smaller RPVs would predict worse outcomes in nephropathy.

Renal mass or volume was supposed to decline with age just as glomerular number and volume would (10). However, kidney mass or volume does not change until 50 or 60 years of age $(9,11,12)$, and kidney mass and number of glomeruli decline with age after 60 years of age. As a result, this decline cannot be entirely explained by age-related glomerulosclerosis in subjects without renal disease (21). In this study, kidney length and RPV were not related to age because relatively young patients with mean glomerulosclerosis rates of around $20 \%$ due to nephropathy were included. Kidney volume best correlated with BSA, whereas kidney length correlated with height in healthy subjects (22). This correlation was also evident in patients with nephropathy.

We discovered that RPV was a useful parameter for estimating eGFR in patients with nephropathy and suggest that nephron damage such as glomerulosclerosis is a possible pathophysiological mechanism to explain the link between eGFR and RPV in patients with nephropathy. In autopsy cases without a history of renal disease, mean glomerular volume and number of glomeruli increased with kidney weight, and both matched well with kidney volume (10). Kidney volume was associated with BSA $(2,5,10,11,21,23)$, even after adjustment for the influence of age. Volume of glomeruli was correlated to BSA, whereas number of glomeruli was not $(10,24)$. Other factors need to be considered for the change in glomeruli volume. were the environmental factors and gene-environment interactions (24). Age and sex-matched African Americans and native West Africans in Senegal, who are presumed to have the same ancestry, have similar total nephron number per kidney. However, mean glomerular volume was greater in African Americans, which could not be completely accounted for differences in body size (25). Larger glomerular size was observed in African Americans who had the same range of body size as the Senegalese (24). Furthermore, an increase in glomerular volume was evident only in African Americans (24). High dietary protein intake also contributed to the enlargement of glomerular volume (26). The glomerular volume in subjects with the same number of glomeruli may change owning to genetic and environmental factors, high dietary protein intake and others. Researchers have proved the relationship between glomerular volume and pathological findings $(21,25)$. Mean glomerular volume was increased in IgA nephropathy with more severe glomerulosclerosis and tubular atrophy, and was estimated based on BMI and glomerular density (number of non-sclerotic glomeruli per renal cortical area in the biopsy samples) in the $\operatorname{IgA}$ nephropathy and non-glomerulonephritis groups (25). Hypertension and CKD were associated with greater mean glomerular volume $(21,27)$. However, the kidney volumes in patients with renal disease may not increase. Although the remaining glomeruli may be enlarged to satisfy the metabolic demands, some nephrons may be damaged and lost. Therefore, glomerular volume may change due to metabolic demands, genetic-environmental interactions, and pathological injuries. RPV in terms of kidney volume may be reflected by glomerular volume and numbers of glomeruli. Previous studies did not investigate changes of kidney volume based on changes in glomerular volume and number of glomeruli, and changes in glomerular volume and number of glomeruli may be due to pathological harm in patients with renal disease. Thus, we investigated the efficacy of kidney volume on estimating pathological findings and outcomes in kidney diseases. In our study, pathologic changes of nephrons were used as link between kidney morphology and eGFR, and between kidney morphology and outcomes.

In patients with renal diseases, some nephrons may be damaged and lose function. Single-nephron glomerular hyperfiltration in remnant glomeruli due to functional nephron loss would lead to progressive glomerular hypertrophy and glomerulosclerosis $(28,29)$. An inverse relationship between RPV and percent of global glomerulosclerosis was also investigated in our study. In patients with atherosclerotic renal artery stenosis, a higher ratio of RPV to GFR would guarantee a better residual renal function after revascularization, which consequently implied RPV as an indicator of 'hibernating' kidney under ischemic condition (12). The relationship between RPV at renal biopsy and the composite outcome in this study could be explained in a similar manner.

This study had limitations. First, it did not have a prospective design and therefore might have selection and data biases. However, the UPCR, serum creatinine, and urinalysis data were collected at renal biopsy in all the patients, and all the data analyzed were complete. The same radiologist and pathologist were involved throughout the study period. However, the data were collected over a period of 5 years and intra-person bias might be present within the data. Secondly, we did not measure kidney morphological parameters in a healthy population by using 3-D USG and did not collect nationwide data on normal range of RPV and kidney length for Koreans. We adopted the RPV and kidney length USG data for a population without renal diseases reported by other investigators and used these as reference for evaluating an exposed group to evaluate the risk of the study outcome. The data would have selection or investigator bias. In order to ensure the validity of our results, we also use a receiver-operator curve to identify the best cut off (mean RPV, $120.7 \mathrm{ml}$ ) in our cohort and used this cutoff for the Kaplan-Meier and Cox regression analysis. However, the results of Cox regression analysis also indicated that the mean RPV of $<125 \mathrm{ml}$, other than the mean RPV of $<120.7 \mathrm{ml}$ $(\mathrm{P}=0.882)$, was a risk factor for the composite outcome (Hazard ratio: $4.287,95 \% \mathrm{CI}$ : $1.133-16.227, \mathrm{P}=0.032$ ) in a fully-adjusted model. Third, diabetic kidney disease and amyloidosis may not show atrophy even under eGFR decline. Thus, $125 \mathrm{ml}$ RPV 
may not be suitable as a reference value for evaluating the risk of outcome in subjects with diabetic and amyloidosis nephropathy. Three patients had diabetic nephropathy and another 3 had amyloidosis in our study. The mean RPV of $<125 \mathrm{ml}$ increased the risk of composite outcome by 4.287 -fold $(95 \%$ CI: 1.133-16.227) compared with the mean RPV of $\geq 125 \mathrm{ml}$. By contrast, the mean RPV of $<125 \mathrm{ml}$ increased the risk of composite outcome by 4.493 -fold (95\% CI: $0.901-22.406)$ as compared with the mean RPV of $\geq 125 \mathrm{ml}$ after exclusion of diabetic and amyloidosis nephropathy. In the 3 diabetic or amyloidosis nephropathy, we observed a trend of higher RPV associated with longer follow-up duration. However, the cases of diabetic and amyloidosis nephropathy were inadequate to analyze the relationship between RPV and the outcome in diabetic and amyloidosis nephropathy. In conclusion, our study shows that smaller RPVs would predict worse outcomes in nephropathy. Further research on diabetic and amyloidosis nephropathy is need. Finally, we did not examine 3-D USG data of patients who underwent renal biopsy within 3 months after onset of symptoms by history-taking to exclude acute change of kidney morphology due to interstitial edema, and water and sodium retention.

In conclusion, kidney morphology is inversely related to nephronal damage and positively related to eGFR in nephropathy diagnosed by using renal biopsy. In the future, whether RPV is an important indicator to guide decision-making during treatment of nephropathies and to estimate renal outcomes of each type of nephropathy should be investigated.

\section{Acknowledgements}

H.J.C. and S.I.H. designed and conducted the research. H.J.L and S.I.H. participated in the conduct of the research and data collection. J.X. analyzed the data and wrote the manuscript and H.J. C. assumes primary responsibility for the final content.

\section{References}

1. Shin HS, Chung BH, Lee SE, Kim WJ, Ha HI and Yang CW: Measurement of kidney volume with multi-detector computed tomography scanning in young Korean. Yonsei Med J 50: 262-265, 2009

2. Sanusi AA, Arogundade FA, Famurewa OC, Akintomide AO, Soyinka FO, Ojo OE and Akinsola A: Relationship of ultrasonographically determined kidney volume with measured GFR, calculated creatinine clearance and other parameters in chronic kidney disease (CKD). Nephrol Dial Transplant 24: 1690-1694, 2009.

3. Jovanović D, Gasic B, Pavlovic S and Naumovic R: Correlation of kidney size with kidney function and anthropometric parameters in healthy subjects and patients with chronic kidney diseases. Ren Fail 35: 896-900, 2013

4. Woodard T, Sigurdsson S, Gotal JD, Torjesen AA, Inker LA, Aspelund T, Eiriksdottir G, Gudnason V, Harris TB, Launer LJ, et al: Segmental kidney volumes measured by dynamic contrast-enhanced magnetic resonance imaging and their association with CKD in older people. Am J Kidney Dis 65: 41-48, 2015.

5. Johnson S, Rishi R, Andone A, Khawandi W, Al-Said J, Gletsu-Miller N, Lin E, Baumgarten DA and O'Neill WC: Determinants and functional significance of renal parenchymal volume in adults. Clin J Am Soc Nephrol 6: 70-76, 2011.

6. Jeon HG, Gong IH, Hwang JH, Choi DK, Lee SR and Park DS: Prognostic significance of preoperative kidney volume for predicting renal function in renal cell carcinoma patients receiving a radical or partial nephrectomy. BJU Int 109 : 1468-1473, 2012.
7. Funahashi Y, Hattori R, Yamamoto T, Aoki S, Majima T and Gotoh M: Renal parenchymal volume increases after contralateral nephrectomy: Assessment using three-dimensional ultrasonography. Int J Urol 18: 857-860, 2011.

8. Cheung CM, Chrysochou C, Shurrab AE, Buckley DL, Cowie A and Kalra PA: Effects of renal volume and single-kidney glomerular filtration rate on renal functional outcome in atherosclerotic renal artery stenosis. Nephrol Dial Transplant 25: 1133-1140, 2010.

9. Griffiths GJ, Cartwright G and McLachlan MF: Estimation of renal size from radiographs: Is the effort worthwhile? Clin Radiol 26: 249-256, 1975.

10. Nyengaard $\mathbf{J}$ and Bendtsen T: Glomerular number and size in relation to age, kidney weight, and body surface in normal man. Anat Rec 232: 194-201, 1992.

11. Gong IH, Hwang J, Choi DK, Lee SR, Hong YK, Hong JY, Park DS and Jeon HG: Relationship among total kidney volume, renal function and age. J Urol 187: 344-349, 2012.

12. Cheong B, Muthupillai R, Rubin MF and Flamm SD: Normal values for renal length and volume as measured by magnetic resonance imaging. Clin J Am Soc Nephrol 2: 38-45, 2007.

13. Kim HC, Yang DM, Jin W and Lee SH: Relation between total renal volume and renal function: Usefulness of 3D sonographic measurements with a matrix array transducer. Am J Roentgenol 194: W186-W192, 2010.

14. Mosteller RD: Simplified calculation of body-surface area. N Engl J Med 317: 1098, 1987.

15. Levey AS, Stevens LA, Schmid CH, Zhang YL, Castro AF III, Feldman HI, Kusek JW, Eggers P, Van Lente F, Greene T, et al: A new equation to estimate glomerular filtration rate. Ann Int Med 150: 604-612, 2009.

16. Lee BH, Ahn HJ, Kang WH And Sho KH: Estimation of kidney size by ultrasonography in normal Korean adults. Korean J Nephrol 18: 46-51, 1999 .

17. Oh SW, Kim S, Na KY, Chae DW, Kim S, Jin DC and Chin HJ: Clinical implications of pathologic diagnosis and classification for diabetic nephropathy. Diabetes Res Clin Pract 97: 418-424, 2012.

18. Partik BL, Stadler A, Schamp S, Koller A, Voracek M, Heinz G and Helbich TH: 3D versus 2D ultrasound: Accuracy of volume measurement in human cadaver kidneys. Invest Radiol 37: 489-495, 2002.

19. Bakker J, Olree M, Kaatee R, de Lange EE and Beek FJ: In vitro measurement of kidney size: Comparison of ultrasonography and MRI. Ultrasound Med Biol 24: 683-688, 1998.

20. Gilja OH, Smievoll AI, Thune N, Matre K, Hausken T, Odegaard S and Berstad A: In vivo comparison of 3D ultrasonography and magnetic resonance imaging in volume estimation of human kidneys. Ultrasound Med Biol 21: 25-32, 1995.

21. Hoy WE, Bertram JF, Denton RD, Zimanyi M, Samuel T and Hughson MD: Nephron number, glomerular volume, renal disease and hypertension. Curr Opin Nephrol Hypertens 17: 258-265, 2008.

22. Emamian SA, Nielsen MB, Pedersen JF and Ytte L: Kidney dimensions at sonography: Correlation with age, sex, and habitus in 665 adult volunteers. AJR Am J Roentgenol 160: 83-86, 1993.

23. Thakur V, Watkins T, McCarthy K, Beidl T, Underwood N, Barnes K and Cook ME: Is kidney length a good predictor of kidney volume? Am J Med Sci 313: 85-89, 1997.

24. McNamara BJ, Diouf B, Douglas-Denton RN, Hughson MD, Hoy WE and Bertram JF: A comparison of nephron number, glomerular volume and kidney weight in Senegalese Africans and African Americans. Nephrol Dial Transplant 25: 1514-1520, 2010.

25. Tsuboi N, Utsunomiya Y,Koike K, Kanzaki G, Hirano K, Okonogi H, Miyazaki Y, Ogura M, Joh K, Kawamura T and Hosoya T: Factors related to the glomerular size in renal biopsies of chronic kidney disease patients. Clin Nephrol 79: 277-284, 2013.

26. Schrijvers BF, Rasch R, Tilton RG and Flyvbjerg A: High protein-induced glomerular hypertrophy is vascular endothelial growth factor-dependent. Kidney Int 61: 1600-1604, 2002.

27. Puelles VG, Zimanyi MA, Samuel T, Hughson MD, Douglas-Denton RN, Bertram JF and Armitage JA: Estimating individual glomerular volume in the human kidney: Clinical perspectives. Nephrol Dial Transplant 27: 1880-1888, 2012.

28. Kandasamy Y, Smith R, Wright IM and Lumbers ER: Relationships between glomerular filtration rate and kidney volume in low-birth-weight neonates. J Nephrol 26: 894-898, 2013.

29. Brenner BM: Nephron adaptation to renal injury or ablation. Am J Physiol 249: F324-F337, 1985. 